\title{
Commentary: Health-Associated Niche Inhabitants as Oral Probiotics: The Case of Streptococcus dentisani
}

\author{
Georg Conrads*, Julia A. Bockwoldt, Caroline Kniebs and Mohamed M. H. Abdelbary \\ Division of Oral Microbiology and Immunology, Department of Operative and Preventive Dentistry and Periodontology, RWTH \\ Aachen University Hospital, Aachen, Germany
}

Keywords: Streptococcus oralis subspecies dentisani, probiotics, dental caries, pH buffering, arginolytic pathway, PCR detection

\section{A commentary on}

Health-Associated Niche Inhabitants as Oral Probiotics: The Case of Streptococcus dentisani by López-López, A., Camelo-Castillo, A., Ferrer, M. D., Simon-Soro, Á., and Mira, A. (2017). Front. Microbiol. 8:379. doi: 10.3389/fmicb.2017.00379

We comment on López-López et al. and their contributions on Streptococcus dentisani, especially the species-specific primers, buffering capacity, and prevalence in the oral cavity of healthy individuals (Camelo-Castillo et al., 2014; López-López et al., 2017), and its infective potential. S. dentisani was effectively reclassified as Streptococcus oralis subspecies dentisani by Jensen et al.

\section{OPEN ACCESS}

Edited by:

Giovanna Batoni,

University of Pisa, Italy

Reviewed by:

Marcelle Nascimento, University of Florida Health,

United States

*Correspondence:

Georg Conrads

gconrads@ukaachen.de

Specialty section:

This article was submitted to

Infectious Diseases,

a section of the journal

Frontiers in Microbiology

Received: 16 November 2017 Accepted: 12 February 2018 Published: 27 February 2018

Citation:

Conrads G, Bockwoldt JA, Kniebs C and Abdelbary MMH (2018)

Commentary: Health-Associated

Niche Inhabitants as Oral Probiotics: The Case of Streptococcus dentisani.

Front. Microbiol. 9:340

doi: 10.3389/fmicb.2018.00340 (2016) and there exist two other closely related subspecies, S. oralis ssp. oralis and S. oralis ssp. tigurinus (Zbinden et al., 2012; Jensen et al., 2016; Conrads et al., 2017). López-López et al. studied the capacity of strains 7746 and $7747^{\mathrm{T}}$ for buffering low $\mathrm{pH}$ and thus preventing overgrowth of acidogenic and aciduric cariogenic species such as Streptococcus mutans. In addition, they developed two PCRs to quantify $S$. oralis ssp. dentisani in clinical specimens, one directed against the carbamate kinase gene $\operatorname{arcC}$ and one against ORF540, the latter coding for a bacteriocin-related protein. Primer specificity was evaluated with DNA from S. mutans, S. sobrinus, S. sanguinis, S. salivarius, S. mitis, S. pneumoniae, S. infantis, and S. oralis ATCC $35037^{\mathrm{T}}$. They conclude that $\operatorname{arcC}$-primers did not amplify any of the related streptococcal species and ORF540-primers cross-reacted with S. pneumoniae only.

With the intention of screening our own strain collection for the presence of $S$. oralis ssp. dentisani, we tested these PCR primers with the type strains of S. oralis ssp. dentisani $7747^{\mathrm{T}}$, ssp. oralis ATCC $35037^{\mathrm{T}}$, ssp. tigurinus $\mathrm{Az3a} \mathrm{a}^{\mathrm{T}}$, six S. mitis strains (OMI 181-184, 187188 ) and four S. pneumoniae strains (OMI 157-159, 186). The arcC-directed PCR (Figure 1A, $\mathrm{CkSd}-\mathrm{F} / \mathrm{R}$ ) was indeed very specific but the product was very short (76 bp) and we designed the alternative CkSdAlt-F/R primer pair amplifying a longer fragment of $175 \mathrm{bp}$ (Figure 1A). The ORF540-directed PCR was found to be more bacteriocin-type than species-directed. Furthermore, we designed a 16S rRNA-gene-directed PCR expanding the options for application (Figure 1A, SDent-16S-F/R). Applying the two Ck- and the 16S-directed PCR, we screened our strain collection of clade S. oralis strains. We found four S. oralis ssp. dentisani strains (SN39325, SN54787, SN54788, SN58364) among isolates of proven and epidemiologically unrelated cases of infective endocarditis (National Reference Center for Streptococci, RWTH Aachen University Hospital, Germany; described in Conrads et al., 2017), and two strains (OMI 214 and OMI 215) isolated from the tooth surface of healthy caries-free probands. Next, we characterized these strains genetically by sequencing the 16S-rRNA gene (Figure 1B) and phenotypically in-vitro for their buffering capacity (Figure 1C). The buffering phenotype is dependent on the arginine deiminase system, which is determined by $\operatorname{arc} A$ (arginine deiminase), $\operatorname{arcB}$ (ornithine carbamoyltransferase), and $\operatorname{arcC}$ 
(carbamate kinase). The system is activated by a $\mathrm{pH}$ drop and leads to ammonia formation and neutralization of the environment and is-besides bacteriocin production - the reason for the probiotic and anti-cariogenic nature. López-López et al. tested the buffer capacity of strain 7746 only under aerobic conditions (A. Mira, personal communication). However, the

A

\begin{tabular}{|c|c|c|c|c|}
\hline Primer & Sequence $5^{\prime}-3^{\prime}$ & $\begin{array}{l}\text { Size } \\
\text { amplicon }\end{array}$ & $\begin{array}{l}\mathrm{Tm} \\
{\left[{ }^{\circ} \mathrm{C}\right]}\end{array}$ & $\begin{array}{l}\mathrm{Ta} \\
{\left[{ }^{\circ} \mathrm{C}\right]}\end{array}$ \\
\hline $\begin{array}{l}\text { CkSd-F } \\
\text { CkSd-R }\end{array}$ & $\begin{array}{l}\text { GTAACCAACCGCCCAGAAGG } \\
\text { CCGCTTTCGGACTCGATCA }\end{array}$ & 76 bp & $\begin{array}{l}62.6 \\
61.3\end{array}$ & 65 \\
\hline $\begin{array}{l}\text { CkSdAltF } \\
\text { CkSdAltR }\end{array}$ & $\begin{array}{l}\text { AATGTGCTCTTGATAACGCATTRC } \\
\text { GGTTGCTCCTGTIIITCGCTC }\end{array}$ & $175 \mathrm{bp}$ & $\begin{array}{l}59.2 \\
62.0\end{array}$ & 62 \\
\hline $\begin{array}{l}\text { SDent-16S-F } \\
\text { SDent-165-R }\end{array}$ & $\begin{array}{l}\text { TGAAGGAGGAGCTTGCTTCTC } \\
\text { CAAACAGTTATCATGCAATAACTG }\end{array}$ & $137 \mathrm{bp}$ & $\begin{array}{l}61.4 \\
56.0\end{array}$ & 59 \\
\hline
\end{tabular}

B

S.pneumoniae ATCC $33400^{T}$

2. S.mitis ATCC 49456

S.pseudopneumoniae ATCC BAA-960'

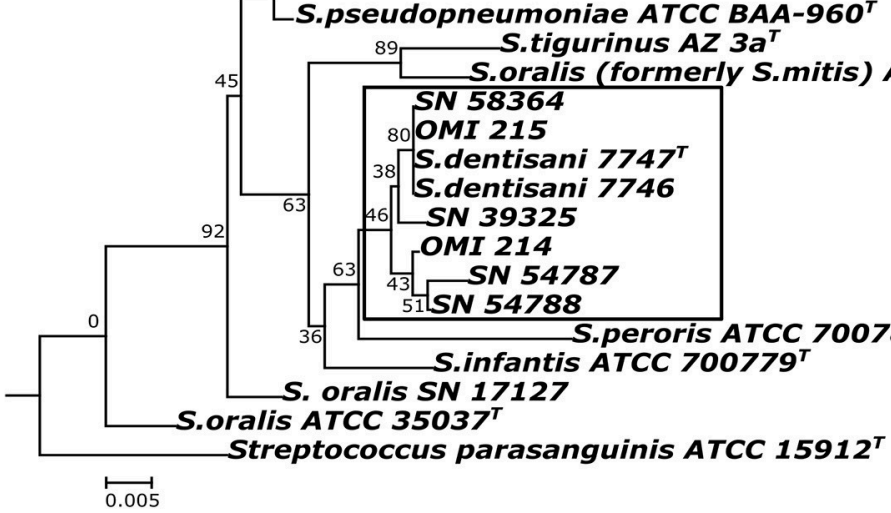

C

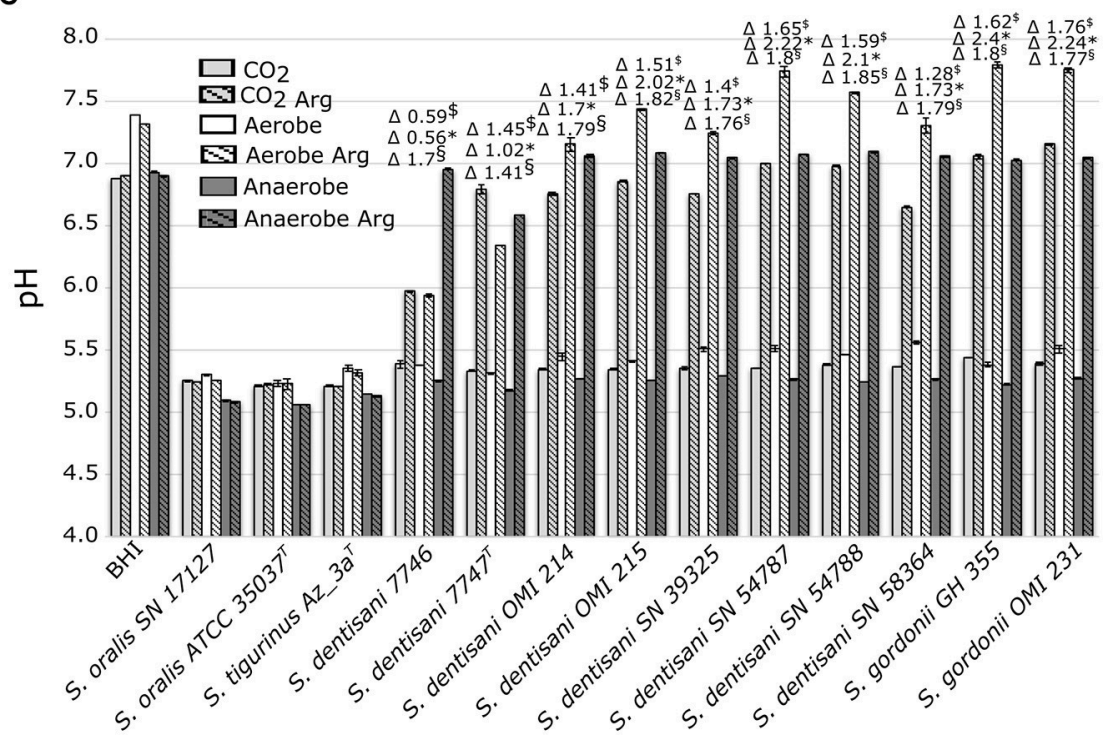

FIGURE 1 | (A) Primer and conditions to specifically amplify S. oralis subspecies dentisani. (B) Neighbor-Joining phylogenetic tree based on the $16 \mathrm{~S}$ rRNA-gene of 17 Mitis group streptococci including strains subjected in this study using MEGA6 with default settings and 200 bootstraps. SN-strains are endocarditis isolates of our collection. S. parasanguinis ATCC $15912^{\top}$ was used as outgroup. (C) Endpoint pH values and $\Delta \mathrm{pH}$ values of S. oralis subspecies dentisani and related (sub-)species after growth over $16 \mathrm{~h}$ in $\mathrm{BHI}$ with and without the addition of arginine monohydrate (Arg, $5 \mathrm{gl}^{-1}$ ) and under $7 \% \mathrm{CO}_{2}$, aerobic and anaerobic conditions. ${ }^{\$} \Delta \mathrm{pH} 7 \%$ $\mathrm{CO}_{2},{ }^{\star} \Delta \mathrm{pH}$ aerobic, ${ }^{\S} \Delta \mathrm{pH}$ anaerobic, BHI, Brain Heart Infusion medium control. The three $\mathrm{S}$. oralis subspecies are abbreviated as $\mathrm{S}$. dentisani, $\mathrm{S}$. oralis, and S. tigurinus. 
atmospheric conditions in the oral cavity and especially on tooth surfaces are rarely truly aerobic, but instead something between micro-aerobic, $\mathrm{CO}_{2}$-rich, and anaerobic. Thus, we reproduced the growth-experiments in the same arginine-enriched BHImedium and applying the same protocol but this time under aerobic, $\mathrm{CO}_{2}$-rich (7\%), and anaerobic conditions. Together with our six $S$. oralis ssp. dentisani strains, we used S. oralis ssp. dentisani $\left(7746,7747^{\mathrm{T}}\right.$ ) and S. gordonii strains (OMI 231, GH 355 ) as positive controls of a functional arginine deiminase system. S. oralis ssp. oralis and ssp. tigurinus, as well as un-inoculated BHI (plus/minus arginine), served as negative controls. Clearly, all our six isolates demonstrated buffering activity, even more strongly pronounced than with the strains 7746 and $7747^{\mathrm{T}}$ (Figure 1C). An increase of buffering activity was detected for $S$. oralis ssp. dentisani strains from $\mathrm{CO}_{2}(\Delta \mathrm{pH} 0.59-$ 1.65 , mean 1.36 , median 1.43$)$ to aerobic $(\Delta \mathrm{pH} 0.56-2.22$, mean 1.64 , median 1.73$)$ to anaerobic $(\Delta \mathrm{pH} 1.41-1.85$, mean 1.74 , median 1.79) conditions. This increased activity under anaerobic conditions was significant for 5 of the 8 S. oralis ssp. dentisani strains tested. In time-curve experiments we confirmed that the alkalization process in arginine-supplemented BHI medium started between 6 and $8 \mathrm{~h}$ after inoculation but in the atmosphere of $7 \% \mathrm{CO}_{2}$ it needed a $\mathrm{pH}$ as low as $\leq 5.5$ to be induced. This finding has clinical impact since under microaerophilic, $\mathrm{CO}_{2}$-rich conditions, re-alkalization might start later than demineralization.

Finally, applying the $\operatorname{arcC}$ - and 16S-subspecies specific primers for real-time PCR-quantification, we subjected 10 saliva samples $(1.5 \mathrm{ml}$ freshly stimulated saliva processed to 100 $\mu l$ DNA extract) of healthy, caries-free probands (aged 2332, randomly selected from an ethically approved past study (Conrads et al., 2017). In principal, the $\operatorname{arcC}$ - and 16S-directed primers led to the same cell numbers which were between $1.2 \times$ $10^{1}$ and $1.2 \times 10^{5}$ cells per $\mu$ l DNA extract or $8.0 \times 10^{2}$ and 8.1 $\times 10^{6}$ per $\mathrm{ml}$ saliva. López-López et al. described much higher numbers $\left(1.04 \times 10^{7}\right.$ and $\left.6.94 \times 10^{7}\right)$ but they were derived

\section{REFERENCES}

Camelo-Castillo, A., Benitez-Paez, A., Belda-Ferre, P., Cabrera-Rubio, R., and Mira, A. (2014). Streptococcus dentisani sp. nov., a novel member of the mitis group. Int. J. Syst. Evol. Microbiol. 64, 60-65. doi: 10.1099/ijs.0.054098-0

Conrads, G., Barth, S., Möckel, M., Lenz, L., van der Linden, M., and Henne, K. (2017). Streptococcus tigurinus is frequent among gtfR-negative Streptococcus oralis isolates and in the human oral cavity, but highly virulent strains are uncommon. J. Oral Microbiol. 9:1307079. doi: 10.1080/20002297.2017.1307079

Jensen, A., Scholz, C. F., and Kilian, M. (2016). Re-evaluation of the taxonomy of the Mitis group of the genus Streptococcus based on whole genome phylogenetic analyses, and proposed reclassification of Streptococcus dentisani as Streptococcus oralis subsp. dentisani comb. nov., Streptococcus tigurinus as Streptococcus oralis subsp. tigurinus comb. nov., and Streptococcus oligofermentans as a later synonym of Streptococcus cristatus. Int. J. Syst. Evol. Microbiol. 66, 4803-4820. doi: 10.1099/ijsem.0.001433

López-López, A., Camelo-Castillo, A., Ferrer, M. D., Simon-Soro, Á., and Mira, A. (2017). Health-associated niche inhabitants as oral probiotics: the case of Streptococcus dentisani. Front. Microbiol. 8:379. doi: 10.3389/fmicb.2017. 00379

Nadkarni, M. A., Martin, F. E., Jacques, N. A., and Hunter, N. (2002). Determination of bacterial load by real-time PCR using a broad-range from the pooled dental plaque of two individuals. Combined with the results of a universal bacterial 16S-directed PCR (modified from Nadkarni et al., 2002), we calculated the relative numbers of $S$. oralis ssp. dentisani within our saliva samples as between 0.01 and $10.46 \%$ (mean $1.73 \%$, median $0.13 \%$ ).

In conclusion, we support the findings of López-López et al. but with the following limitations: (i) the ORF450-directed primer showed low accuracy. We have designed alternative primers amplifying a longer $\operatorname{arcC}$-fragment and a V1-V2fragment of the 16S, expanding the applications; (ii) the induction and potential of alkalization of $S$. oralis ssp. dentisani is dependent on the atmosphere, with best results obtained under anaerobic conditions; (iii) as we found four endocarditisassociated strains in our collection and a Danish group recently even found six (Rasmussen et al., 2017), it must be indicated that some strains of $S$. oralis ssp. dentisani might bear the potential for causing infective endocarditis in immunocompromised patients. The challenge thus is to find-or produce-a strain with the most probiotic and the least infective potential.

\section{AUTHOR CONTRIBUTIONS}

GC supervised the study, wrote the commentary, and is the corresponding author. JB performed experiments on the phenotypic characterization of $S$. dentisani isolates. CK performed experiments on the genotypic characterization of $S$. dentisani isolates and designed the PCR primers. MA checked genomic data and all results for plausibility and commented on and corrected the manuscript.

\section{ACKNOWLEDGMENTS}

We wish to thank Mark van der Linden (National Reference Center for Streptococci, RWTH Aachen University Hospital, Aachen, Germany) for providing the IE-related strains and Mrs. Beate Melzer for her excellent technical assistance.

(universal) probe and primers set. Microbiology 148, 257-266. doi: 10.1099/00221287-148-1-257

Rasmussen, L. H., Højholt, K., Dargis, R., Christensen, J. J., Skovgaard, O., Justesen, U. S., et al. (2017). In silico assessment of virulence factors in strains of Streptococcus oralis and Streptococcus mitis isolated from patients with infective endocarditis. J. Med. Microbiol. 66, 1316-1323. doi: 10.1099/jmm.0.000573

Zbinden, A., Mueller, N. J., Tarr, P. E., Spröer, C., Keller, P. M., and Bloemberg, G. V. (2012). Streptococcus tigurinus sp. nov., isolated from blood of patients with endocarditis, meningitis and spondylodiscitis. Int. J. Syst. Evol. Microbiol. 62, 2941-2945. doi: 10.1099/ijs.0.038299-0

Conflict of Interest Statement: The authors declare that the research was conducted in the absence of any commercial or financial relationships that could be construed as a potential conflict of interest.

Copyright (C) 2018 Conrads, Bockwoldt, Kniebs and Abdelbary. This is an open-access article distributed under the terms of the Creative Commons Attribution License (CC $B Y)$. The use, distribution or reproduction in other forums is permitted, provided the original author(s) and the copyright owner are credited and that the original publication in this journal is cited, in accordance with accepted academic practice. No use, distribution or reproduction is permitted which does not comply with these terms. 